\title{
Overexpression of the Panax ginseng MYB4 gene enhances stress tolerance in transgenic Arabidopsis thaliana
}

\author{
W.H. LIAN ${ }^{1}$, T.X. SUN ${ }^{1}$, X.Y. MENG ${ }^{2}$, R. SUN ${ }^{1}$, F. HUI ${ }^{1}$, Y.N. JIANG ${ }^{1, *}$, and Y. ZHAO ${ }^{1, *}$ \\ ${ }^{1}$ Jilin Ginseng Academy, Changchun University of Chinese Medicine, Changchun 130117, P.R. China \\ ${ }^{2}$ Jilin Yatai Bio-pharmaceuticals Co. Ltd, Changchun 130000, P.R. China \\ *Corresponding authors: E-mail: cnzhaoyu1972@126.com,ynjiangsyl@163.com
}

\begin{abstract}
The myeloblastosis (MYB) transcription factors are essential for plant stress responses. They can enhance plant tolerance to abiotic stresses (e.g., drought, salinity, and cold) via improved physiological and biochemical responses including the accumulation of metabolites. In this study, we constructed a Panax ginseng MYB4 (PgMYB4) gene expression vector and established the stable transgenic Arabidopsis thaliana lines to study the effects of this gene on plant stress tolerance. The germination rate and seedling taproot length were greater for the $P g M Y B 4$-overexpressing plants than for the wild-type plants. Accordingly, the overexpression of PgMYB4 in Arabidopsis enhanced seedling tolerance to drought, salt, and cold conditions. Under drought stress, the relative chlorophyll content decreased less, the proline content increased more, and the water loss rate decreased more in the transgenic plants than in the wild type. The expressions of stress-related genes responsive to dehydration $19 \mathrm{~A}$, responsive to dehydration 22 , responsive to desiccation $29 \mathrm{~A}$, cold-regulated 15A, cold-regulated 47, and pyrroline-5-carboxylate synthase 1 were significantly upregulated in the transgenic Arabidopsis plants. Under high salt stress, the kinesin 1 (KIN1) expression was significantly upregulated in the transgenic plants. In response to the low temperature stress, the dehydration-responsive element binding protein $2 \mathrm{~A}$ and KIN1 expressions increased dramatically in the transgenic Arabidopsis plants. Thus, PgMYB4 positively regulated the stress tolerance gene networks, which promoted the expression of anti-stress effector genes. This gene may be useful for ginseng breeding programs aiming to develop new cultivars with enhanced stress tolerance.
\end{abstract}

Keywords: cold stress, drought, gene expression, salinity.

\section{Introduction}

The MYB gene family, which is one of the largest transcription factor (TF) families in plants, plays a vital role in plant growth and responses to environmental stresses. The first $M Y B$ gene (v-Myb proto-oncogene) was discovered in the avian myeloblastic tumor virus genome (Klepnauer et al. 1982). Homologs of the $M Y B$ gene were subsequently identified in vertebrates, insects, and fungi (Lipsick et al. 1996, Weston et al. 1998). Of the $M Y B$ homologs in plants, $M Y B C 1$ in maize, was the first identified (Paz-Ares et al. 1987), with a function related to pigment synthesis. Many $M Y B$ genes have been isolated and identified in plants (Marocco et al. 1989, Stracke et al. 2001, Du et al. 2013, Yamagishi et al. 2020, Yuan et al. 2020), and there is considerable interest in functionally characterizing these genes. A highly conserved DNA binding domain defines the MYB TFs at the $\mathrm{N}$-terminus (Du et al. 2012). The binding domain contains 1 - 4 imperfect repeating sequences (Rs), each $\mathrm{R}$ segment comprises approximately 52 amino acids residues and includes three regularly spaced tryptophan residues, which together

$\overline{\text { Received }} 6$ May 2020, last revision 8 November 2020, accepted 11 November 2020.

Abbreviations: ABA - abscisic acid; COR15A - cold-regulated 15A; COR47 - cold-regulated 47; DREB2A - dehydration-responsive element binding protein 2A; KIN1 - kinesin-1; P5CR - pyrroline-5-carboxylate reductase; P5CS1 - pyrroline-5-carboxylate synthase 1; RD19A - responsive to dehydration 19A; RD22 - responsive to dehydration 22; RD29A - responsive to desiccation 29A; TF transcription factor.

Acknowledgements: This work was supported by the Ministry of Science and Technology (2018YFC1706603, 2017YFC1702102) and the Jilin Province Science and Technology Development Plan (20170309007YY). We thank Margaret J. Sporck-Koehler, Ph.D., from Liwen Bianji, Edanz Editing China, for editing the English text of a draft of this manuscript.

Conflict of interest: The authors declare that they have no conflict of interest. 
form three $\alpha$-helices. The second and third $\alpha$-helices of each repeat fold into a helix-turn-helix structure, and the third helix of each repeat directly intercalates in the major groove of DNA (Chen et al. 2006). The MYB TF family contains four main subfamilies, which are grouped by the number of tandem repeats they possess: 1RMYB/ MYB-related, R2R3-MYB, R1R2R3-MYB, and 4R-MYB (Dubos et al. 2010, Zhao et al. 2019). Among these groups, the R2R3-MYB subfamilies are the most common in plant MYBs (Stracke et al. 2001).

The R2R3-MYB TFs are involved in various physiological and biological processes, such as secondary metabolism, cellularmorphogenesis, cellulardifferentiation, and cell-cycle control (Du et al. 2009, Gujjar et al. 2014). Some also play essential roles in responses to abiotic stresses (Cao et al. 2013). In A. thaliana, some R2R3-MYB genes, AtMYB44 TF regulates ABA-mediated stomatal closure, which confers abiotic stress tolerance (Jung et al. 2008); while the AtMYB15 TF is involved in the regulation of $C$-repeat binding factor genes under cold stress, and it plays a role in enhanced freezing tolerance (Agarwal et al. 2006). The ectopic expression of rice Osmyb4 in Arabidopsis results in the upregulated expression of the pyrroline-5-carboxylate synthetase gene P5CS1, which increases proline content and improves the tolerance of the transgenic Arabidopsis plant to drought stress (Mattana et al. 2005). The CsMYB0 and CsMYB2 proteins from Cucumis sativus are involved in the responsiveness to ABA signalling, high salinity, and low temperature $(\mathrm{Li}$ et al. 2012). In Gossypium hirsutum, GhMYB108-like is an important regulatory gene in response to drought and salt stresses (Ullah et al. 2020). The BplMYB46 TF from Betula platyphylla was reported to improve salt and osmotic stress tolerance. The BplMYB46 TF inhibits the expression of proline degradation genes, which can result in elevated proline content and improved abiotic stress tolerance (Guo et al. 2017). In a recent study involving an in vitro ginseng root tissue culture model, PgMYB1 gene transcription increased by up to 4-fold in response to cold or high salinity (Afrin et al. 2015). Although MYB TFs are well studied, their functional roles are not entirely understood. Furthermore, many members of the MYB TF family are still not described.

Ginseng is one of the primary medicinal plants in East Asia. Some of the medical benefits of ginseng include helping to balance metabolic activities, protecting the nervous system, and enhancing the endocrine system (Yue et al. 2007, Cho et al. 2014). However, the cultivation of ginseng usually requires 3 - 20 years. Its growth and development are greatly affected by irradiance, water, soil, temperature, and other environmental conditions. Among environmental factors, drought, high salinity, and low temperatures are the main abiotic stresses that limit ginseng yield and quality (Wu et al. 2005, Lee et al. 2019). In this study, we isolated a new ginseng $M Y B T F$, named $M Y B 4$, and investigated whether it is important for abiotic stress responses by overexpressing ginseng MYB4 in Arabidopsis. Furthermore, we examined the mechanism by which ginseng MYB TFs improve plant tolerance to drought, high salt, and freezing conditions.

\section{Materials and methods}

Plants and cultivation: We collected five-year-old Panax ginseng C.A Meyer plants from Fusong County in Jilin Province, which is the leading commercial ginsengproducing region in China. We placed Arabidopsis thaliana L. (Columbia-0 ecotype) seeds on half-strength Murashige and Skoog (MS) medium in Petri plates. Plants were cultivated in an artificial climate growth chamber at a temperature of $22{ }^{\circ} \mathrm{C}$, a 16 -h photoperiod, and an irradiance of $100 \mu \mathrm{mol} \mathrm{m} \mathrm{m}^{-2} \mathrm{~s}^{-1}$.

Extraction of RNA and cDNA synthesis: We used liquid nitrogen to homogenize the ginseng roots to a fine powder, and we isolated the total RNA with the TRIzol reagent (Invitrogen; Thermo Fisher Scientific, Waltham, MA, USA), according to an improved TRIzol method (Wolfe-Simon et al. 2006). We examined the RNA purity and concentration using a spectrophotometer (Shimadzu, Kyoto, Japan). We used electrophoresis on $1 \%(\mathrm{~m} / \mathrm{v})$ agarose gel to determine the quality of the RNA. We performed reverse transcription using a TaKaRa reverse transcription kit (Takara Biotechnology Co., Dalian, China), and the first strand of cDNA was synthesized according to the manufacturer's protocol and stored at $-80{ }^{\circ} \mathrm{C}$ for further use.

Cloning PgMYB4 cDNA: We used the reverse transcription-polymerase chain reaction (RT-PCR) to clone the PgMYB4 cDNA. The PgMYB4 gene sequence was obtained from the ginseng transcriptome database (http://blast.ncbi.nlm.nih.gov/Blast.cgi, PRJNA659400), after which gene-specific primers were designed with the PRIMER 5.0 software (MYB4-F: TGCTCTAGAATGGTGAGAGCTCCTTGCTGTG; MYB4-R: CGCGGATCCGAATTCAGGTAAGTCCC CAGCT). The Beijing Genomics Institute (BGI) synthesized the primers. We performed the PCR reaction under the following program: initial denaturation at $94{ }^{\circ} \mathrm{C}$ for $2 \mathrm{~min}$, then 30 cycles of denaturation at $94{ }^{\circ} \mathrm{C}$ for $30 \mathrm{~s}$, annealing at $60{ }^{\circ} \mathrm{C}$ for $30 \mathrm{~s}$, and elongation at $72{ }^{\circ} \mathrm{C}$ for $1 \mathrm{~min}$, followed by a 5 -min extension at $72^{\circ} \mathrm{C}$. The total volume of the PCR reaction mixture was $50 \mathrm{~mm}^{3}$. The mixture contained $10 \mathrm{~mm}^{3}$ of $5 \times \mathrm{PCR}$ buffer, $28.75 \mathrm{~mm}^{3}$ of $\mathrm{ddH}_{2} \mathrm{O}, 0.25 \mathrm{~mm}^{3}$ of TaKaRa Ex Taq ${ }^{\circledR}$ Hot Start Version, $0.5 \mathrm{~mm}^{3}$ of each primer, $10 \mathrm{~mm}^{3}$ of cDNA template. We selected the specific PCR product using electrophoresis on $2 \%$ agarose gel.

Construction of a plant expression vector and transformation of Arabidopsis plants: The PCR products were linked to the pMDTM18-T vector (Takara, Dalian, China). After digestion with BamHI and $\mathrm{XbaI}$, the specific DNA fragment was incorporated into the pCAMBIA1303 binary plant expression vector (Beijing Dingguo Changsheng Biotechnology Co., Beijing, China), which contains a CaMV $35 \mathrm{~S}$ promoter and NOS terminator. We sequenced the recombinant plasmids at the BGI and analyzed with the DNAMAN software.

The recombinant pCAMBIA1303 plasmid carrying 
PgMYB4 was inserted into Agrobacterium tumefaciens strain Ag10, and then used to infect Arabidopsis plants according to a floral dip method (Clough et al. 1998). We screened the transgenic plants with $60 \mathrm{mg} \mathrm{dm}^{-3}$ hygromycin in half-strength MS medium. Then, we tested the seeds with hygromycin from the putative PgMYB4-carrying transgenic plants for another two generations to obtain T3 transgenic Arabidopsis plants. The RT-qPCR confirmed the expression of PgMYB4 in the T3 generation of plants.

Analysis of the PgMYB4 gene: We used the DNAMAN software to perform the gene sequence analysis of the PgMYB4 protein. We identified the homology of PgMYB4 using protein $B L A S T$ in the $N C B I$ database (http://blast. ncbi.nlm.nih.gov/Blast.cgi) and conducted a phylogenetic analysis using the MEGA 7.0 program and the neighborjoining method. The strength of nodes in the tree was set by bootstrap analysis (1 000 replicates). We used the website ExPASy (http://www.expasy.org) to analyze the relative molecular mass and isoelectric point.

Analyses of RNA and protein: Total RNA was extracted from the transgenic Arabidopsis plants according to an established cetyltrimethyl ammonium bromide (CTAB) method ( $\mathrm{Hu}$ et al. 2002), after which cDNA was synthesized by RT-PCR. The transcriptions of several abiotic stress tolerance genes (i.e., responsive to dehydration $19 A$ (RD19A), responsive to dehydration 22 (RD22), responsive to desiccation $29 A$ (RD29A), coldregulated 15A (COR15A), cold-regulated 47 (COR47), and pyrroline-5-carboxylate synthase 1 (P5CS1), dehydrationresponsive element binding protein $2 A(D R E B 2 A)$, and kinesin-1 (KIN1) were analyzed by RT-qPCR, with Arabidopsis thaliana ubiquitin as a reference gene. Details regarding the PCR primers are provided in Table 1 Suppl. We examined the protein extracts by sodium dodecyl sulphate-polyacrylamide gel electrophoresis. A Western blot was performed with a polyclonal rabbit antiserum against a PgMYB4 peptide (CDNSGDTTDFNRGA) as the primary antibody and AP-labeled goat anti-rabbit IgG $(1: 1000 ; \mathrm{v} / \mathrm{v})$ as the secondary antibody. We combined nitro blue tetrazolium chloride and 5-bromo-4-chloro-3indolyl phosphate to produce protein bands, and we took images of the bands.

Drought tolerance: Arabidopsis seedlings were grown for 4 weeks and then they were not supplied with water until more than $50 \%$ of the plants showed varying degrees of wilting. After rehydrating the plants, we calculated their survival rates. Samples were collected before and after the drought stress treatment and immediately frozen in liquid nitrogen for an RT-qPCR analysis. The drought-induced gene transcriptions were measured.

The chlorophyll content of seedling leaves at similar positions on plants was determined with a portable chlorophyll meter (SPAD-502, KONICA MINOLT, Tokyo, Japan) on the $5^{\text {th }}$ day of drought stress. At the same time, the free proline content was determined as previously described (Bates et al. 1973).

Plants were grown for 4 weeks, after which the shoots were collected. Under a constant temperature $\left(25 \pm 1{ }^{\circ} \mathrm{C}\right)$ and a humidity $(60 \%)$, the samples were weighed every $20 \mathrm{~min}$ up to $180 \mathrm{~min}$. This analysis was completed with three replicates, each with three plants. The average water loss rate of the leaves was calculated based on the fresh mass basis.

Osmotic stress tolerance: Four-week-old seedlings were irrigated with 100,200 , or $300 \mathrm{mM} \mathrm{NaCl}$ every $4 \mathrm{~d}$, and then they were watered normally for 1 week, after which phenotypic changes were observed. Samples were collected before and after the salt stress treatment and immediately frozen in liquid nitrogen for an RT-qPCR analysis.

Arabidopsis seeds were placed on half-strength MS medium containing various concentrations of $\mathrm{NaCl}(100$, 200 , or $300 \mathrm{mM})$ or mannitol $(100,200$, or $300 \mathrm{mM})$. After $3 \mathrm{~d}$ of vernalization at $4{ }^{\circ} \mathrm{C}$, the seeds were incubated in an artificial climate growth chamber under an irradiance of $100 \mu \mathrm{mol} \mathrm{m} \mathrm{m}^{-2} \mathrm{~s}^{-1}$ for $7 \mathrm{~d}$. Seeds were considered to be germinated if sprouting was detected (at least $1 \mathrm{~mm}$ long).

Arabidopsis seeds were placed on normal halfstrength MS medium in Petri plates and vernalized at $4{ }^{\circ} \mathrm{C}$ for $3 \mathrm{~d}$. The plates were then placed vertically in an artificial climate growth chamber under irradiance of $100 \mu \mathrm{mol} \mathrm{m} \mathrm{m}^{-2} \mathrm{~s}^{-1}$ for $5 \mathrm{~d}$. After the roots reached $1 \mathrm{~cm}$ in length, the seedlings were transferred to half-strength MS medium containing various concentrations of $\mathrm{NaCl}$ or mannitol. The root lengths were measured after $11 \mathrm{~d}$. All the stress treatments were performed with independent three biological replicates.

Freezing tolerance: Healthy transgenic plants were incubated at $-6^{\circ} \mathrm{C}$, and then they recovered for one week, after which the phenotypic changes were observed. Samples were collected before and after the low temperature stress treatment and immediately frozen in liquid nitrogen for a RT-qPCR analysis.

Statistical analysis: Statistical analyses were performed using the SPSS software (SPSS Inc., Chicago, IL, USA). We analyzed the significance level, and we considered differences between means to be significant and extremely significant when $P$ values were less than 0.05 and 0.01 , respectively.

\section{Results}

The $P g M Y B 4$ gene was cloned from ginseng roots by RTPCR. The total length of the PgMYB4 gene was $735 \mathrm{bp}$, and it encoded 245 amino acid (AA) with an isoelectric point of 5.41 (Fig. 1 Suppl.). The predicted molecular mass of the PgMYB4 protein was $27.9 \mathrm{kDa}$. The $P g M Y B 4$ gene sequence was accessed in the GenBank (https://www. ncbi.nlm.nih.gov/genbank, ID MN998542).

Next, we generated transgenic Arabidopsis thaliana plants carrying PgMYB4 gene under the control of the CaMV 35S promoter. PCR analysis of the putative transgenic plants confirmed the amplification of an 

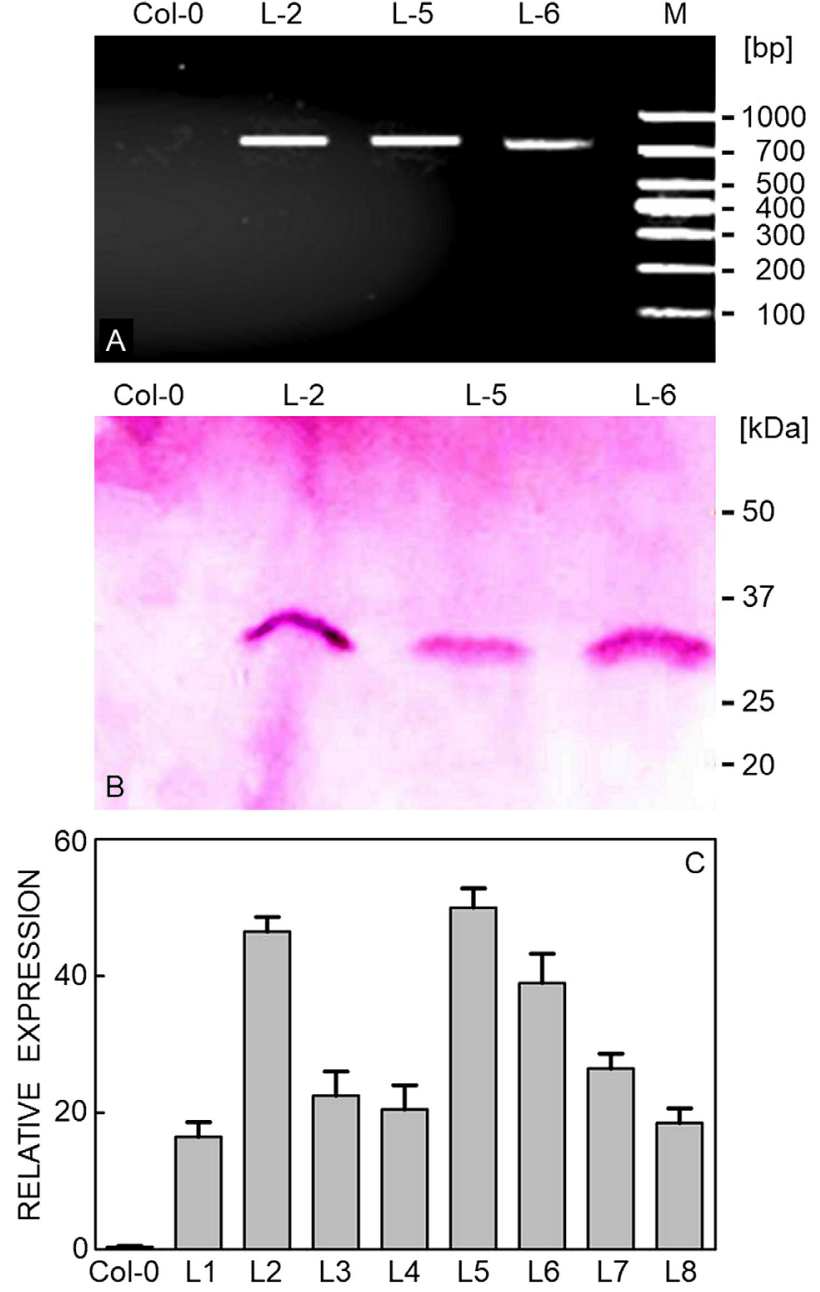

Fig. 1. Analysis by PCR $(A)$ and Western-blot detection $(B)$ of transgenic Arabidopsis plants. The expression of Panax ginseng myeloblastosis 4 (PgMYB4) in different transgenic Arabidopsis lines $(C)$. Col-0 - wild-type Arabidopsis, L1 to L8 - transgenic Arabidopsis lines, M - DL 1000TM DNA marker.

approximately 730 -bp fragment in the $\mathrm{T} 3$ generation plants resistant to hygromycin (Fig. 1A). The same fragment was not amplified in the wild type Arabidopsis (control). Consistent with the PCR results, Western blot involving anti-PgMYB4 polyclonal antibodies confirmed that the transgenic Arabidopsis plants, but not the wild-type plants, produced a protein between 25 and $37 \mathrm{kDa}$ (Fig. 1B). The expression of PgMYB4 in transgenic Arabidopsis was detected in all PgMYB4 transgenic lines but not in the wild type (Fig. 1C). Three independent transgenic lines (L2, L5, and L6) showed significantly higher expression than others, and we selected them for subsequent experiments.

The NCBI database comparative analysis showed that PgMYB4 contained two highly conserved MYB domains, which were located at 14 - 63 AA and 67 - 114 AA, respectively (Fig. 2 Suppl.). The PgMYB4 protein is consistent with the conserved domain of the R2R3MYB subfamily. For further analysis, we used the NCBI $B L A S T$ tool to conduct the multiple sequence alignment of PgMYB4 with the MYBs from other plants (Fig. $2 A$ ). The PgMYB4 gene exhibits a high homology with the Cucumis sativus MYB4 (XP_008447428.1), Fragaria vesca MYB4 (XP 004151985.1), and Gossypium hirsutum MYB4 (XP 016709539.1). Like other Panax ginseng MYB proteins, PgMYB4 contains two domains. Each domain has three tryptophan residues that are spaced by 18 or 19 AAs. However, in the R3 MYB4 motif, the first tryptophan residue is replaced by phenylalanine (Afrin et al. 2015, Choi et al. 2017). The result shows that PgMYB4 belongs to the R2R3-MYB subfamily.

To further identify $P g M Y B 4$ gene in $P$. ginseng, we constructed a phylogenetic tree (Fig. 2B). The phylogenetic analysis involved PgMYB4 with 11 MYB AA sequences from other species. Our results showed that PgMYB4 was most closely related to Cucumis sativus and Fragaria vesca, followed by Gossypium hirsutum. Interestingly, the majority of R2R3-MYB proteins, similar to PgMYB4, seems to be involved in response to various biotic and abiotic stresses (He et al. 2016). Therefore, we can speculate that PgMYB4 protein might be involved in abiotic stress responses.

We evaluated the osmotic stress tolerance of the PgMYB4-overexpressing transgenic Arabidopsis plants by placing $\mathrm{T}_{3}$ transgenic seeds in half-strength MS medium containing various mannitol concentrations. The germination rate of the transgenic plants (L2, L5, and L6) was significantly higher than that of the wild-type plants for all three tested concentrations. The differences between the transgenic and wild-type plants were more apparent as the mannitol concentration increased. At $300 \mathrm{mM}$ mannitol, the germination rates of the transgenic plants were $51.78,49.13$, and $47.72 \%$, respectively, whereas the germination rates of wild-type plants were only $25 \%$ (Fig. 3A). Conversely, the germination rate for both plant types was $100 \%$ in the normal culture medium.

The root length of transgenic and wild-type Arabidopsis plants was also carried on seedlings grown in the mannitolcontaining medium for $11 \mathrm{~d}$. The roots of the transgenic Arabidopsis were longer than those of the wild-type Arabidopsis. The difference in the root lengths increased with increasing mannitol concentrations. Specifically, the root lengths of the transgenic Arabidopsis plants were about 1.3-fold and 2.1-fold greater than those of the wildtype plants at mannitol concentrations of $200 \mathrm{mM}$ and 300 $\mathrm{mM}$, respectively (Fig. 3B,C). There were no differences in the primary root length between transgenic and wildtype plants in the control culture medium.

The overexpression of PgMYB4 improved the survival rate of whole plants exposed to severe water shortage. We did not supply water to the $\mathrm{T}_{3}$ generation plants for $14 \mathrm{~d}$. No obvious phenotypic differences were detected between the transgenic and wild-type plants on day 5. However, on day 14, the wild-type plants were yellowish and wilted, and some plants died, whereas the transgenic plants exhibited less severe dehydration stress symptoms (e.g., fewer yellow leaves) (Fig. 4A). After re-watering for $7 \mathrm{~d}$, the survival rates of the transgenic and wild-type plants were $80,75,78$, and $30 \%$, respectively (Fig. 4B). The much higher recovery rate for the $P g M Y B 4$-overexpressing 
A

Panax ginseng

Cucumis sativus Gossypium hirsutum

Fragaria vesca

Betula platyphylla

consensus

Panax ginseng

Cucumis sativus

Gossypium hirsutum

Fragaria vesca

Betula platyphylla

consensus

Panax ginseng

Cucumis sativus

Gossypium hirsutum

Fragaria vesca

Betula platyphylla

consensus

Panax ginseng

Cucumis sativus

Gossypium hirsutum

Fragaria vesca

Betula platyphylla consensus

Panax ginseng

Cucumis sativus

Gossypium hirsutum

Fragaria vesca

Betula platyphylla consensus
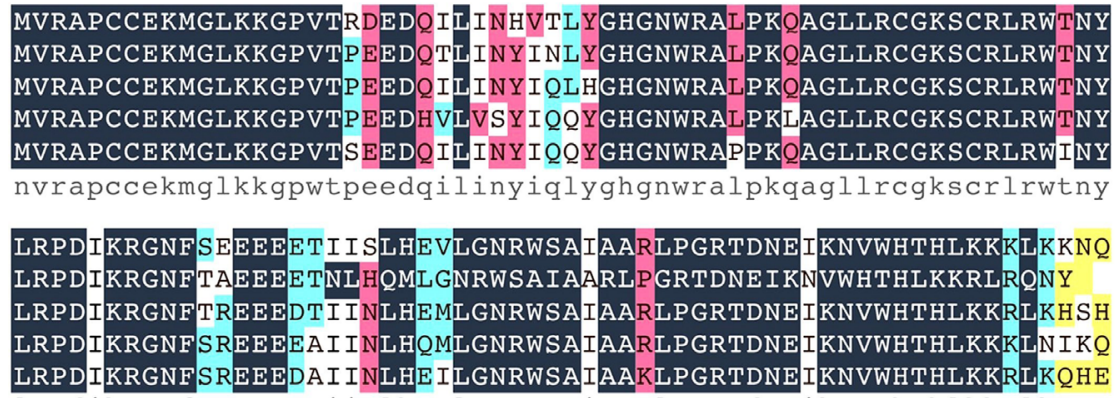

lrpdikrgnfsreeeetiinlhemlgnrwsaiaarlpgrtdneiknvwhthlkkrlkqng

LENSPDQILSKSEP...KPMKQS TTQST....SHDDLPNSPQSITT.......TSSE 163 ATPQLRNYPVMNEEE..SIAQQPFKYTNYG.SMPSSALAFSPPHCSS . . . ESSSIITAE 173 GSNANNRQPIDPSED. IKREQQPVTIYS..........PVSPPQSSSSS . .DVSTS ..EN 163 SPTTPEALGQVSEPELPKPEDASCHAKSE . . . SFEYGPVSPPQCTTSSADDTSSFTTSE 176 ATPVATKRRSKHYQE. AKSELEPMDFPSHENSGSHEHRPVSPQECSS . . . DMSTVTIGD 175 atn $p$ pskseee kpeqqp $t$ s she lpvsppqcis d ss ttse

DNN . . . . . MSIHTAEAF . . DDTFWSELFSTDEN . .SGN . . TSDNSGD. TTDFNRGAQ 208 NGE. . . . . . . SVTNNLPEADENFWREVLGSDQM . . . . . ATVGNQOGFEGETSERQ 215 NSNSH . TFTTNTETNEDVSEIDENFWSEVLSADNS . SMAADFRVVGS.DQYFPSSPPPP 219 NNNSS SANMGMNVDYEVSLPEADESFWSEVLSADHN . . SPNELVSSHFGDQAIGAAENPQ 234 DNNDN . . CTCPKAESPVNF PEVDEDFWSEVLSTES SGVVNDDF SAVGGGDPPLDQVPFSP 233 nnns $n n$ et enfpeadenfwsevlsadn $s$ ndf svgsgdq $d p$ pq

LQIFPEFGCENNLNMNDG. . MDFFWYDILTKSAGDLPEF

LGNLTNE. . . . QFTHET. . . TDFWTIFFQE.EGIYLS

LPALETVNGCG. SNLYDTDANMDFWYNLFTR. AADLPELPE

VNTSMDYGFSG. SNMYEN . . CMDFWYDVFTR. AGEIPELLD

LMTMEPVHGYG. SNMHDS . . MDFWFNVFTR. AGEIPELPD

I tle vgg g snnhdt ndfwydvftr agdipelpd

B

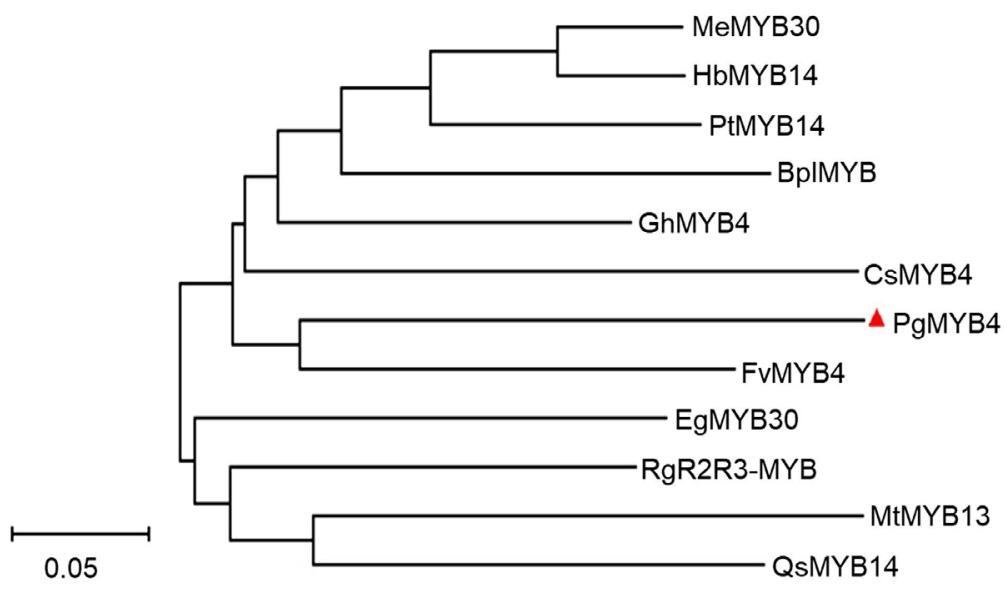

Fig. 2. Sequence analysis of myeloblastosis 4 from Panax ginseng (PgMYB4). A - Sequence alignment of PgMYB4 with other homolog proteins, including CsMYB4 (Cucumis sativus, XP_004151985.1), GhMYB4 (Gossypium hirsutum, XP_016709539.1), FvMYB4 (Fragaria vesca subsp. vesca, XP_004289939.1), BplMYB (Betula platyphylla, QEK22814.1). B - a phylogenetic tree of PgMYB4. The sequences used are from: MeMYB30 (Manihot esculenta, XP_021629490.1), HbMYB14 (Hevea brasiliensis, XP_021656703.1), PtMYB14 (Populus trichocarpa, XP_002302045.1), BplMYB (Betula platyphylla, QEK22814.1), GhMYB4 (Gossypium hirsutum, XP 016709539.1), CsMYB4 (Cucumis sativus, XP 004151985.1), FvMYB4 (Fragaria vesca subsp. vesca, XP 004289939.1), EgM̄YB30 (Elaeis guineensis, XP_010920477.1 ), RgR2R3-MYB (Rehmannia glutinosa, AKV71950.1), MtMYB̄13 (Medicago truncatula, XP_013468731.1), and QsMYB14 (Quercus suber, XP_023879142.1).

transgenic plants implied that PgMYB4 increased the drought tolerance of the transgenic Arabidopsis.

The overexpression of PgMYB4 increased the plant survival rate under drought conditions. Consequently, we examined three physiological indices (i.e., chlorophyll content, proline content, and water loss rate) of the droughtstressed transgenic and wild type Arabidopsis plants. Under normal conditions, the leaf chlorophyll content of the transgenic and wild-type Arabidopsis plants was similar. After a 5-d drought treatment, the leaf chlorophyll 

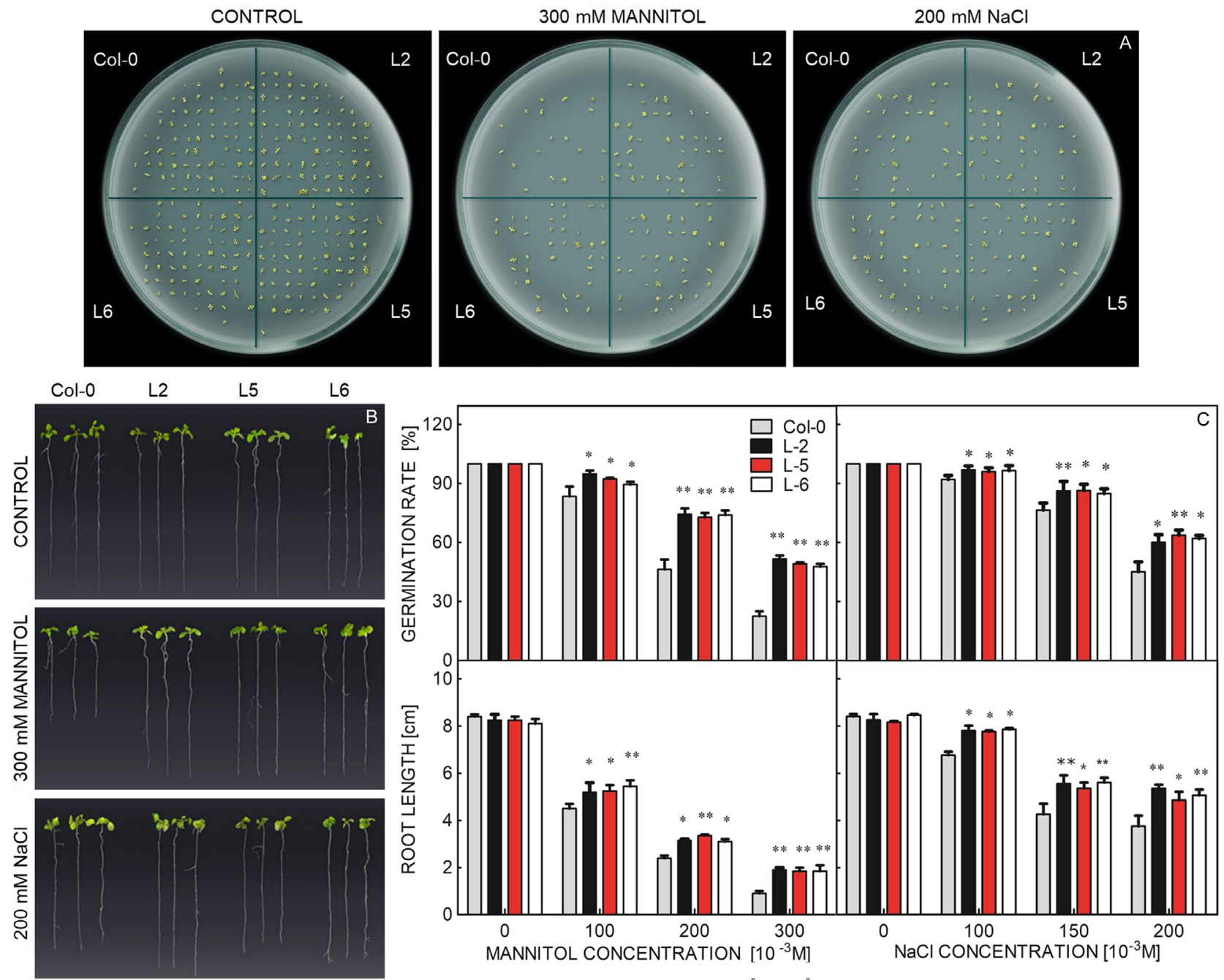

Fig. 3. Effects of abiotic stresses on seed germination and root growth of wild-type Arabidopsis (Col-0) and transgenic Arabidopsis lines (L2, L5, and L6). Seed germination and root growth $(A, B)$, and germination rate and root length $(C)$ under mannitol and $\mathrm{NaCl}$ stresses. Means \pm SDs, $n=3$; ** and * indicate statistically significant differences compared to the control at $P<0.01$ and $P<0.05$, respectively.

content in transgenic Arabidopsis decreased by only 2.80 , 4.81, and $3.78 \%$, which was much less than the $13.85 \%$ decrease in the wild-type Arabidopsis (Fig. 5A). The proline content increased by about 3.3 -fold in transgenic plants, but only 2.2 -fold in wild-type plants (Fig. $5 B$ ). The water loss rates from the shoots of the transgenic and wildtype plants were about $50.13,51.1,51.11$, and $64.73 \%$ at the 3-h time-point (Fig. 5C).

To better understand the possible regulatory mechanisms of PgMYB4 in response to drought stress, the expressions of drought stress-related genes, including RD19A, RD22, RD29A, COR15A, COR47, and P5CS1 (Guo et al. 2014, Qin et al. 2015), was detected in transgenic and wild-type lines by RT-qPCR. Before drought treatment, most of the gene expressions showed no obvious differences between the WT and transgenic plants. However, under drought stress, the expression of five genes was significantly induced (Fig. 6). Specifically, the overexpression of PgMYB4 increased the expression of $R D 19 A$ by 2.7 - to 3 -folds, $R D 22$ by 2.2 to 3.8 -folds,
$R D 29 A$ by 1.9 - to 3.6 -folds, COR $15 A$ by 5.2 - to 6.6 -folds, COR 47 by 2.6 - to 3.6-folds, and P5CS1 2.5- to 4.3 -folds.

Overexpression of PgMYB4 conferred salt stress tolerance to transgenic Arabidopsis plants as the germination rate was higher for the $T_{3}$ transgenic seeds than for the wild-type seeds in half-strength MS medium supplemented with $\mathrm{NaCl}$. The differences were more obvious at higher $\mathrm{NaCl}$ concentrations. At $200 \mathrm{mM} \mathrm{NaCl}$, the germination rates of the transgenic and wild-type seeds were 61 to $63 \%$ and $45 \%$, respectively (Fig. 3C). The overexpression of $P g M Y B 4$ improved the development of the main root under high salt stress, as determined by the root length. Arabidopsis seedlings were exposed to high salt stress for $11 \mathrm{~d}$. The roots of transgenic plants were longer than those of wild-type, with more substantial differences under higher $\mathrm{NaCl}$ concentrations. In medium containing $200 \mathrm{mM} \mathrm{NaCl}$, the transgenic Arabidopsis roots were $4.8-5.6 \mathrm{~cm}$ long, which were 1.3 - to 1.5 -times longer than the wild-type plant roots (Fig. 3C).

Under high salt stress, the whole-plant survival rates 


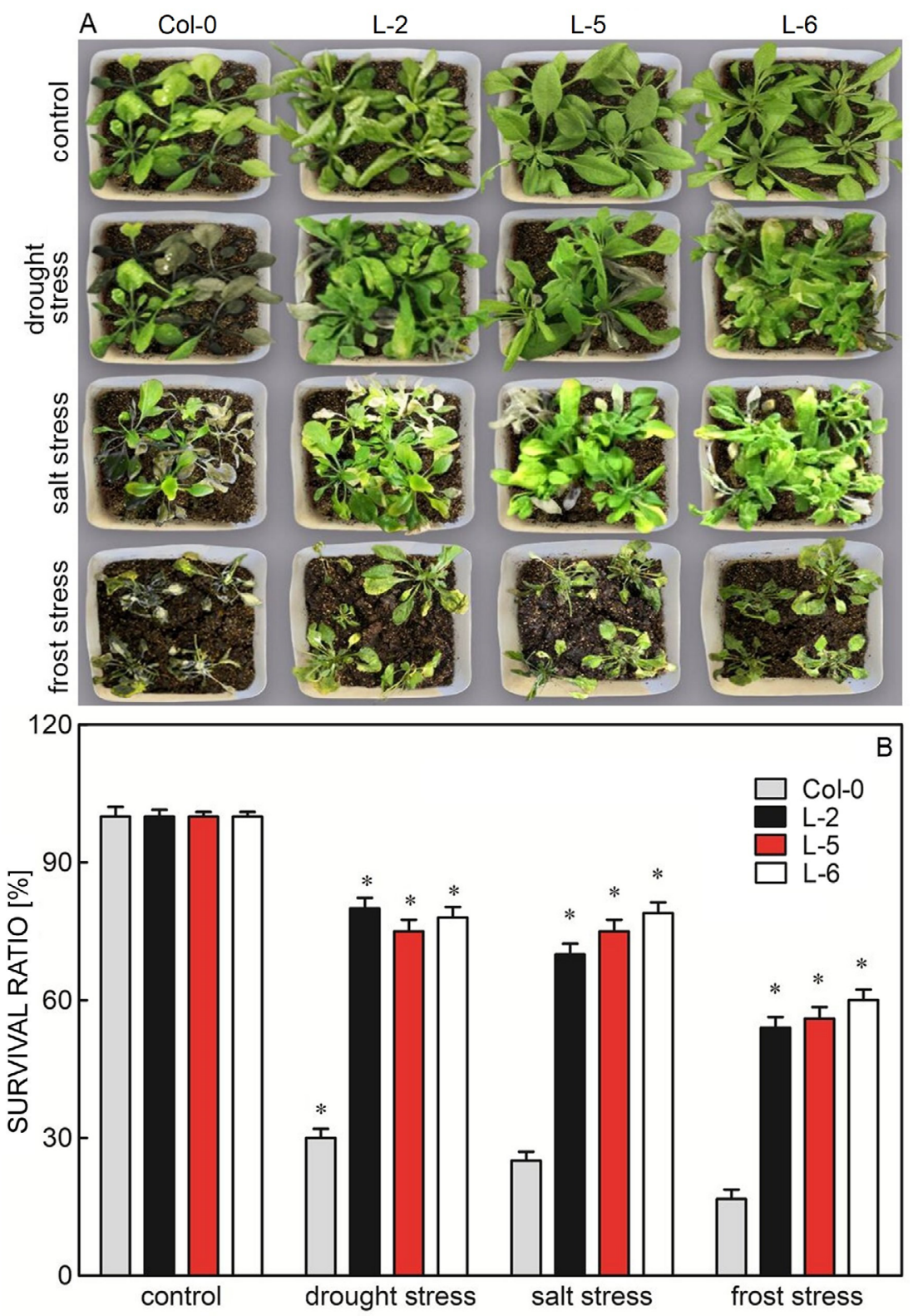

Fig. 4. Responses of transgenic and wild-type Arabidopsis plants to drought, high salinity, and frost stresses. $A$ - phenotypic analysis of transgenic Arabidopsis (L-2, L-5, and L-6) and wild-type plants (Col-0). B - survival rates of transgenic and wild-type plants. Col-0 wild-type Arabidopsis; L2, L5, L6 - transgenic Arabidopsis lines. The total number of surviving plants / total number of plants employed in the experiment for each line: drought stress: L2 (40/50), L5 (39/52), L6 (43/55), Col-0 (16/53); salt stress: L2 (36/50), L5 (39/52), L6 (43/55), Col-0 (13/53); frost stress: L2 (23/50), L5 (25/52), L6 (28/55), Col-0 (8/53). Means \pm SDs, $n=3$; ** and * indicate statistically significant differences compared to the control at $P<0.01$ and $P<0.05$, respectively.

were higher for the PgMYB4-overexpressing plants than for the wild-type controls. When plants were treated with $300 \mathrm{mM} \mathrm{NaCl}$, the leaves of both the transgenic and wildtype plants turned yellow, but to varying degrees. The transgenic Arabidopsis plants grew much better than the wild-type plants. Specifically, the wild-type plants had many yellow leaves and some died. After allowing plants to recover by watering for one week, the survival rate $(72$, 75 , and $78 \%$ ) of transgenic Arabidopsis was about 3-times higher than that $(25 \%)$ of the wild-type control (Fig. $4 B$ ).

To investigate the possible mechanism of the PgMYB4mediated response to salt stress, we analyzed the expressions of salt stress-related genes. Before salt treatment, the genes showed no differences or slightly higher expressions in the transgenic plants. Among all analyzed stress-related genes, the expressions of most genes were not induced significantly under salt-stress, except KIN1 (Fig. 7 and Fig. 3 Suppl.). Its expression increased by 20 -fold in wildtype plants exposed to high salinity. The KIN1 expression in the PgMYB4-overexpressing transgenic plants was 7.8fold greater than that in the wild-type plants (Fig. 7). These results suggested that $P g M Y B 4$ might be involved in the regulation of salt stress response.

After incubation at $-6{ }^{\circ} \mathrm{C}$ for $50 \mathrm{~min}$, the leaves of both 

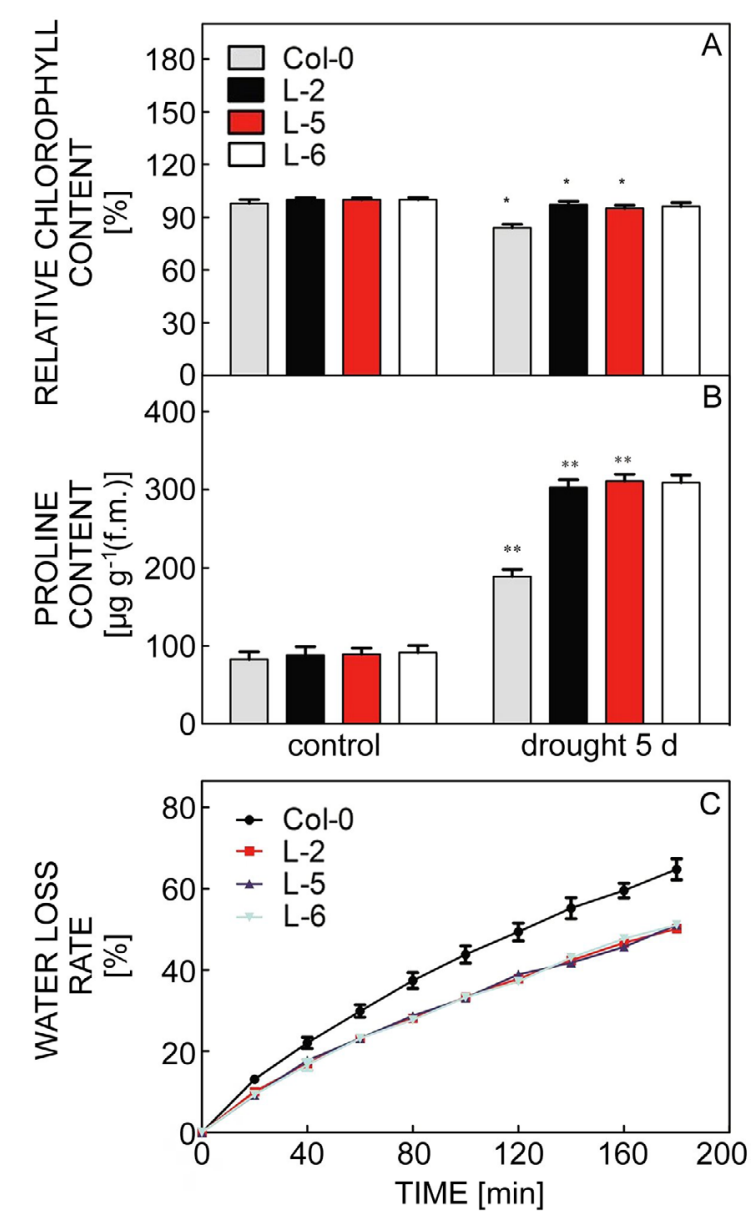

Fig. 5. Relative chlorophyll content $(A)$, proline content $(B)$, and water loss $(C)$ of wild-type and transgenic Arabidopsis under drought stress for 5 d. Col-0 - wild-type Arabidopsis; L2, L5, L6 - transgenic Arabidopsis. Means \pm SDs, $n=3$; ** and * indicate statistically significant differences compared to the control at $P<$ 0.01 and $P<0.05$, respectively.

wild-type and transgenic Arabidopsis plants were dark green (i.e., freezing damaged). After a 1-week recovery, the wild-type leaves were more damaged (i.e., dark green and dehydrated) than the transgenic leaves. The survival rates of the wild-type and transgenic Arabidopsis plants were about $16.7 \%$ and 54 to $60 \%$, respectively (Fig. $4 B$ ).

To further clarify the PgMYB4-mediated increase in the frost tolerance of transgenic Arabidopsis, we analyzed the expressions of stress-related genes in response to low temperature conditions. Obvious expression changes were detected for $D R E B 2 A$ and KIN1. The expressions of other genes decreased. After the freezing treatment, the DREB2A and KIN1 expression were 3.4- to 3.8-times and 2.9- to 3.4-times higher, respectively, in the transgenic plants than in the wild-type Arabidopsis plants (Fig. 7). Thus, the increased resistance of PgMYB4-overexpressing plants to frost stress may result, in part, from the enhanced expression of these two genes.

\section{Discussion}

Stress affects plant growth and development, even though plants generally have a mechanism to overcome various biotic and abiotic stresses. Arabidopsis thaliana is a model plant, which is often used along with transgenic techniques to investigate gene functions related to stress responses (Wu et al. 2018, Yao et al. 2018). In the current study, we constructed an expression vector with the ginseng MYB4 gene for the subsequent transformation of Arabidopsis. We studied if the overexpression of PgMYB4 can increase the abiotic stress tolerance of the transgenic plants.

To investigate whether $P g M Y B 4$ influences stress responses, we assessed the stress tolerance of transgenic Arabidopsis during the seedling stages. Seed germination is dependent on environmental factors, such as water, temperature, and oxygen (Hoang et al. 2014). Abiotic stress restricts germination by altering physiological or biochemical events.

Mannitol and $\mathrm{NaCl}$ can decrease the water absorption by plants by decreasing the substrate osmotic potential (Jakab et al. 2005). Treating the seeds of wild-type and PgMYB4-overexpressing transgenic plants with different mannitol and $\mathrm{NaCl}$ concentrations resulted in decreased germination rates and root lengths. The differences between the wild-type and transgenic plants were greater under higher mannitol or $\mathrm{NaCl}$ concentrations. These observations imply that the transgenic Arabidopsis seeds were able to better absorb or retain water than the wild-type seeds, which likely contributed to their greater germination rate and root growth. These results also indicate that the overexpression of PgMYB4 might significantly increase plant tolerance to drought or high salt stress.

Chlorophyll is critical for photosynthesis in plants and its content directly influences photosynthesis and metabolism. To some extent, the chlorophyll content influences stress tolerance. A severe lack of water damages the structure of chloroplast lamellae and lowers the chlorophyll content of plant leaves. Accordingly, the chlorophyll content may be a useful indicator of plant sensitivity to drought stress (Dong et al. 2019, Carvalho et al. 2019). In this study, we observed that drought stress decreased the chlorophyll content considerably more in the wild type than in the PgMYB4-overexpressing Arabidopsis, which indicates a more substantial drought tolerance of the transgenic plants. Proline is one of the components of plant proteins. Proline accumulates in highly drought-tolerant plants (Vannini et al. 2007). Similar to chlorophyll, proline content can act as a physiological indicator of drought tolerance (Szabados et al. 2010). Our data revealed a greater accumulation of proline in PgMYB4-overexpressing Arabidopsis than in the wild-type controls, indicating that the transgenic plants are more drought-tolerant than the wild-type plants. The water loss rate from the shoots of the transgenic Arabidopsis was slower than from shoots of the wildtype, suggesting that the introduction of PgMYB4 might promote stomatal closure in leaves to minimize water loss, thereby enhancing drought tolerance.

The overexpression of $P g M Y B 4$ increased the tolerance of transgenic Arabidopsis to drought, high salinity, and 


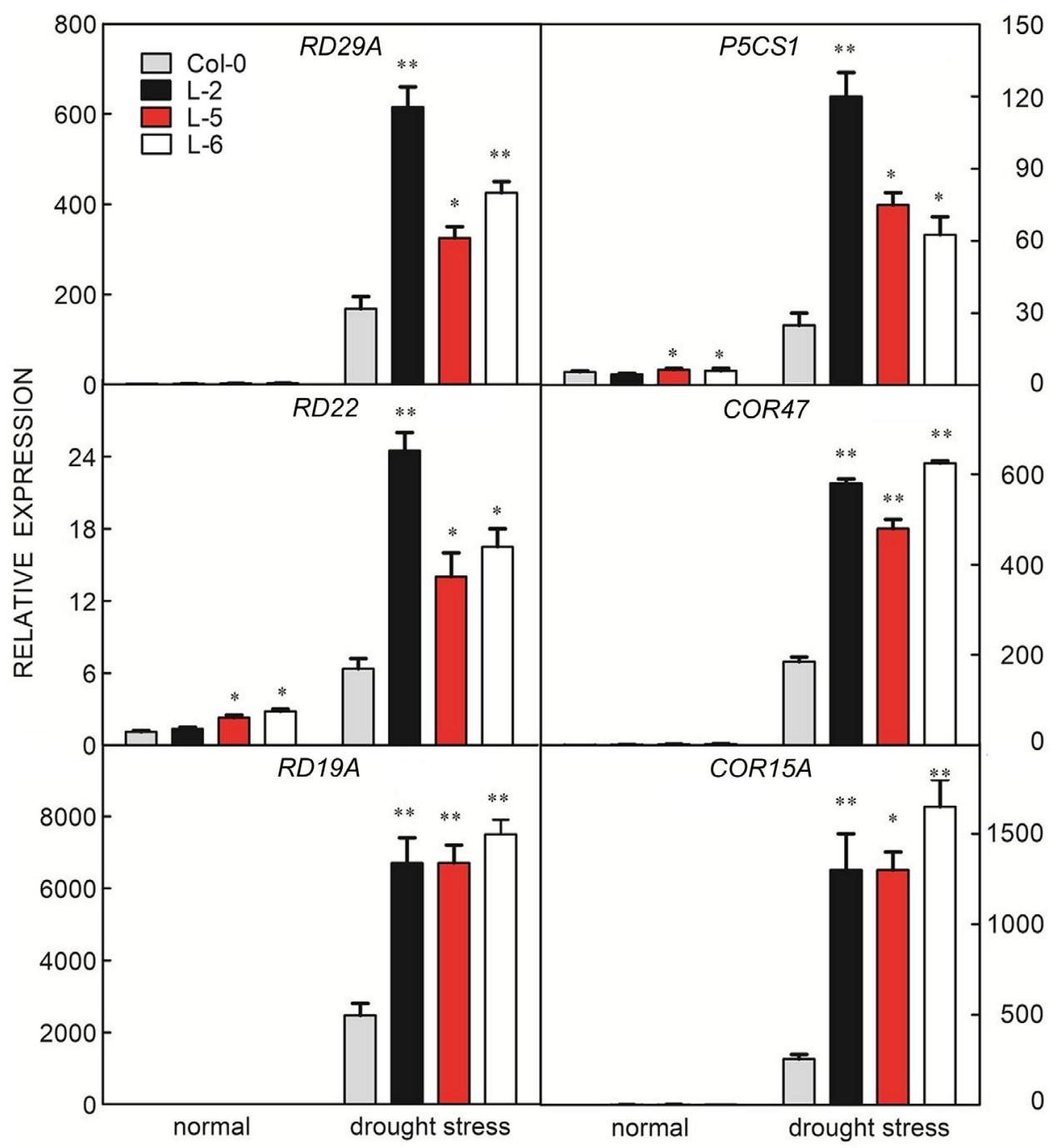

Fig. 6. Expression of drought stress related genes in wild-type and transgenic Arabidopsis under normal conditions and drought stress. Col-0 - wild-type Arabidopsis; L2, L5, L6 - transgenic Arabidopsis. RD29A - responsive to desiccation 29A, P5CS1 - pyrroline-5carboxylate synthase 1, RD22 - responsive to dehydration 22, COR47 - cold-regulated 47, RD19A - responsive to dehydration $19 \mathrm{~A}$, COR15A - cold-regulated $15 A$. Means \pm SDs, $n=3 ; * *$ and * indicate statistically significant differences compared to the control at $P<0.01$ and $P<0.05$, respectively.

low-temperature stresses. Under all three stress conditions, the survival rates of the transgenic Arabidopsis were at least threefold more elevated than the survival rates of the wild-type plants. These findings are consistent with those of previous studies on different MYB TFs. For example, the ectopic expression of maize CmMYB2 in Arabidopsis enhances the tolerance to drought and salt stresses (Shan et al.2012). Additionally, wheat TaMYB33-overexpressing transgenic Arabidopsis plants are reportedly sensitive to ABA and highly tolerant of drought and saline conditions (Qin et al. 2012). Thus, PgMYB4 influences the responses of transgenic Arabidopsis to some abiotic stresses.

To confirm the mechanism underlying this enhanced tolerance to drought, salt, and low-temperature due to $P g M Y B 4$ overexpression, we quantified the expressions of several marker genes associated with stress responses. It is known that RD19A, RD22, RD29A, COR15A, COR47, and P5CS1 are inducible by cold, salt, or drought stress
(Guo et al. 2014, Wang et al. 2016). KIN1 is an important cold-inducible gene in Arabidopsis. DREBs have been involved in cold stress through regulating the transcription of the low temperature responsive genes (Nakashima et al. 2009).

Under non-stress conditions, RD19A, RD29A, COR15A, COR47, P5CS1 and KIN1 showed similarly low expressions in $P g M Y B 4$-overexpressing and wild type plants. However, under drought or salt stress, the expressions of these genes in the transgenic plants were higher than in the wild type plants (Fig. 6 and 7). PgMYB4-overexpression did not enhance the expressions of stress-responsive genes under normal growth condition. We speculate that PgMYB4 may mediate the activation of these stress-responsive genes accompanied by other stressresponsive regulators. Under drought stress, RD19A, RD22, RD29A, COR15A, COR47, and P5CS1 were more highly expressed in the transgenic Arabidopsis than in the 

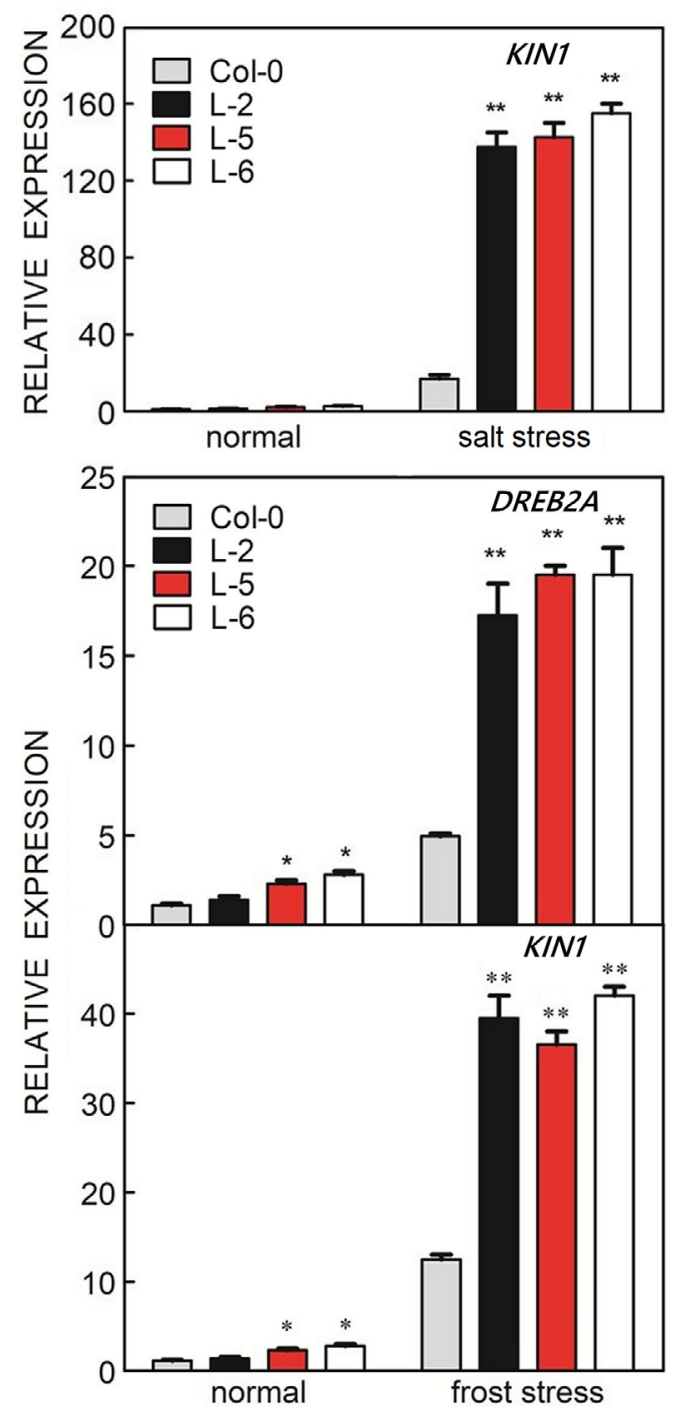

Fig. 7. Expressions of high salt stress and frost stress related genes in transgenic Arabidopsis under normal conditions and under salt or frost stress. Col-0 - wild-type Arabidopsis. L2, L5, L6 - transgenic Arabidopsis. Means $\pm \mathrm{SD}, n=3$; ** and * indicate statistically significant differences compared to the control at $P<$ 0.01 and $P<0.05$, respectively.

wild-type. Drought, which is one of the most common stresses plants are exposed to, affected the expression of more genes compared to the other tested abiotic stresses. In response to the high salt and cold stress, the expression of $K I N 1$ in transgenic plants was up-regulated compared with that in wild type plants. In addition, $D R E B 2 A$ expression is substantially higher in transgenic plants than in wildtype under low temperature conditions (Guo et al. 2014). We observed that the P5CS1 gene was significantly upregulated in transgenic plants under drought conditions. Pyrroline-5-carboxylate synthetase (P5CS) is the crucial enzyme for proline biosynthesis in plants (De Ronde et al. 2004). The increased expression of P5CS1 in transgenic plants resulted in increased proline accumulation. Our findings were consistent with the previous reports that overexpression of P5CS1 gene in transgenic plants resulted in accumulation of free proline, and increased tolerance to abiotic stress (Hong et al. 2016).

Our studies suggest that overexpression of PgMYB4 resulted in drastically increased tolerance to abiotic stresses probably by elevated stress-responsive gene expression and inhibited stomata opening and water loss. These findings imply that PgMYB4 probably functions as a positive regulatory factor that improves stress tolerance by up regulating the expression of multiple stress-related genes, and involved in the mediation of stoma function.

\section{References}

Afrin, S., Zhu, J., Cao, H.Z., Huang, J.J., Xiu, H., Luo, T., Luo, Z.Y.: Molecular cloning and expression profile of an abiotic stress and hormone responsive $M Y B$ transcription factor gene from Panax ginseng. - Acta biochem. biophys. sin. 4: 267277,2015

Agarwal, M., Hao, Y., Kapoor, A., Dong, C.H., Fujii, H., Zheng, X., Zhu, J.K.: A R2R3 type MYB transcription factor is involved in the cold regulation of $C B F$ genes and in acquired freezing tolerance. - J. biol. Chem. 281: 37636-37645, 2006.

Bates, L.S., Waldren, R.P., Teare, I.D.: Rapid determination of free proline for water stress studies. - Plant Soil 39: 205-207, 1973.

Cao, Z.H., Zhang, S.Z., Wang, R.K., Zhang, R.F., Hao, Y.J.: Genome wide analysis of the apple MYB transcription factor family allows the identification of MdoMYB121 gene confering abiotic stress tolerance in plants. - Plos ONE 8: e69955, 2013

Carvalho, M., Castro, I., Moutinho-Pereira J., Correia C., EgeaCortines, M., Matos, M., Rosa, E., Carnide V., Lino-Neto T.: Evaluating stress responses in cowpea under drought stress. - J. Plant Physiol. 241: 153001, 2019.

Chen, Y.H., Yang, X.Y., He, K., Liu, M.H., Li, J.G., Gao, Z.F., Lin, Z.Q., Zhang, Y.F., Wang, X.X., Qiu, X.M., Shen, Y.P., Zhang, L., Deng, X.H., Luo, J.C., Deng, X.W., Chen, J.L., Gu, H.Y., Qu, L.J.: The MYB transcription factor superfamily of Arabidopsis: expression analysis and phylogenetic comparison with the rice MYB family. - Plant mol. Biol. 60: 107-124, 2006.

Cho, H.M., Kang, Y.H., Yoo, H., Yoon, S.Y., Kang, S.W., Chang, E.J., Song, Y.: Panax ginseng red extract regulates energy expenditures by modulating PKA dependent lipid mobilization in adipose tissue. - Biochem. biophys. Res. Commun. 4: 644-648, 2014.

Choi, J.Y., Abbaib, R., Kima, Y.J., Silvab, J., Rahimib, S., Myagmarjavb, D., Chunga, I.S., Kwona, W.S., Yang, D.C.: Molecular characterization of MYB transcription factor genes from Panax ginseng. - Russ. J. Plant Physiol. 64: 398-409, 2017.

Clough, S.J., Bent, A.F.: Floral dip: a simplified method for Agrobacterium-mediated transformation of Arabidopsis thaliana. - Plant J. 16: 735-743, 1998.

De Ronde, J.A., Cress, W.A., Krüger, G.H.J., Strasser, R.J., Van Staden, J.: Photosynthetic response of transgenic soybean plants, containing an Arabidopsis P5CR gene, during heat and drought stress. - J. Plant Physiol. 161: 1211-1224, 2004.

Dong, S.K., Jiang, Y.Z., Dong, Y.C., Wang, L.B., Wang, W.J., Ma, Z.Z., Yan, C., Ma, C.M., Liu, L.J.: A study on soybean responses to drought stress and rehydration. - Saudi. J. biol. Sci. 26: 2006-2017, 2019.

Du, H., Wang, Y.B., Xie, Y., Liang, Z., Jiang, S.J., Zhang, S.S., 
Huang, Y.B., Tang, Y.X.: Genome-wide identification and evolutionary and expression analyses of $M Y B$-related genes in land plants. - DNA Res. 20: 437-448, 2013.

Du, H., Yang, S.S., Liang, Z., Feng, B.R., Liu, L., Huang, Y.B., Tang, Y.X.: Genome-wide analysis of the MYB transcription factor superfamily in soybean. - BMC Plant Biol. 12: 1-22, 2012.

Du, H., Zhang, L., Liu, L., Tang, X.F., Yang, W.J., Wu, Y.M., Huang, Y.B., Tang. Y.X.: Biochemical and molecular characterization of plant MYB transcription factor family. Biochemistry (Moscow) 74: 1-11, 2009.

Dubos, C., Stracke, R., Grotewold, E., Weisshaar, B., Martin, C., Lepiniec, L.: MYB transcription factors in Arabidopsis. Trends Plant Sci. 15: 573-581, 2010.

Gujjar, R.S., Akhtar, M., Singh, M.: Transcription factors in abiotic stress tolerance. Ind. J. Plant Physiol. 19: 306-316, 2014

Guo, H.Y., Wang, Y.C., Wang, L.Q., Hu, P., Wang, Y.M., Jia, Y.Y., Zhang, C.R., Zhang, Y., Zhang, Y.M., Wang, C., Yang, C.P.: Expression of the MYB transcription factor gene BplMYB46 affects abiotic stress tolerance and secondary cell wall deposition in Betula platyphylla. - Plant biotechnol. J. 15: 107-121, 2017.

Guo, W.L., Chen, R.G., Du, X.H., Zhang, Z., Yin, Y.X., Gong, Z.H., Wang, G.Y.: Reduced tolerance to abiotic stress in transgenic Arabidopsis overexpressing a Capsicum annuum multiprotein bridging factor 1. - BMC Plant Biol. 14: 1-13, 2014.

He, Q.L., Jones, D.C., Li, W., Xie, F.L., Ma, J., Sun, R.R., Wang, Q.L., Zhu, S.J., Zhang, B.H.: Genome-wide identification of $R 2 R 3-M Y B$ genes and expression analyses during abiotic stress in Gossypium raimondii. - Sci. Rep. 16: 22980, 2016.

Hoang, H.H., Sechet, J., Bailly, C., Leymarie, J., Corbineau, F.: Inhibition of germination of dormant barley (Hordeum vulgare L.) grains by blue light as related to oxygen and hormonal regulation. - Plant Cell Environ. 37: 1393-1403, 2014.

Hong, Y., Zhang, H., Huang, L., Li, D., Song, F.: Overexpression of a stress-responsive NAC transcription factor gene ONAC022 improves drought and salt tolerance in rice. - Front. Plant Sci. 7: 1-19, 2016

Hu, C.G., Chikako, H., Masayuki, K., Zilian, Z., Tamomi, T., Takaya, M.: A simple protocol for RNA isolation from fruit trees containing high levels of polysaccharides and polyphenol compounds. - Plant mol. Biol. Rep. 1: 69a-69g, 2002.

Jakab, G., Ton, J., Flors, V., Zimmerli, L., Métraux, J.P., Brigitte, M.M.: Enhancing Arabidopsis salt and drought stress tolerance by chemical priming for its abscisic acid responses. - Plant Physiol. 139: 267-274, 2005.

Jung, C., Seo, J.S., Han, S.W., Koo, Y.J., Kim, C.H., Song, S.I., Nahm, B.H., Choi, Y.D., Cheong, J.J.: Overexpression of AtMYB44 enhances stomatal closure to confer abiotic stress tolerance in transgenic Arabidopsis. - Plant Physiol. 146: 623635,2008

Klempnauer, K.H., Gonda, T.J., Bishop, J.M.: Nucleotide sequence of the retroviral leukemia gene $\mathrm{v}$-myb and its cellular progenitor c-myb: the architecture of a transduced oncogene. - Cell 31: 453-463, 1982.

Lee, H.J., Jeong, J., Alves, A.C., Han, S.T., In, G., Kim, E.H., Jeong, W.S., Hong, Y.S.: Metabolomic understanding of intrinsic physiology in Panax ginseng during whole growing seasons. - J. Ginseng Res. 43: 654-665, 2019.

Li, Q., Zhang, C., Li, J., Wang, L., Ren, Z.: Genome-wide identification and characterization of $R 2 R 3 M Y B$ family in Cucumis sativus. - Plos ONE 7: 1-18, 2012.

Lipsick, J.S.: One billion years of MYB. - Oncogene 13: 223235, 1996.
Marocco, A., Wissenbach, M., Becker, D., Paz-Ares, J., Saedler, H., Salamini, F., Rohde, W.: Multiple genes are transcribed in Hordeum vulgare and Zea mays that carry the DNA binding domain of the myb oncoproteins. - Mol. gen. Genet. 216: 183$187,1989$.

Mattana, M., Biazzi, E., Consonni, R., Locatelli, F., Vannini C., Provera, S., Coraggio, I.: Overexpression of Osmyb4 enhances compatible solute accumulation and increases stress tolerance of Arabidopsis thaliana. - Physiol. Plant. 125: 212-223, 2005.

Nakashima, K., Ito, Y., Yamaguchi-Shinozaki, K.: Transcriptional regulatory networks in response to abiotic stresses in Arabidopsis and grasses. - Plant Physiol. 149: 88-95, 2009.

Paz-Ares, J., Ghosal, D., Wienand, U., Peterson, P.A., Saedler, H.: The regulatory $\mathrm{c} 1$ locus of Zea mays encodes a protein with homology to myb proto-oncogene products and with structural similarities to transcriptional activators. - EMBO J. 6: 3553-3558, 1987.

Qin, Y.X., Tian, Y.C., Liu, X.Z.: A wheat salinity-induced WRKY transcription factor TaWRKY93 confers multiple abiotic stress tolerance in Arabidopsis thaliana. - Biochem. biophys. Res. Commun. 464: 428-433, 2015.

Qin, Y.X., Wang, M.C., Tian, Y.C., He, W.X., Han, L., Xia, G.M.: Over-expression of TaMYB33 encoding a novel wheat MYB transcription factor increases salt and drought tolerance in Arabidopsis. - Mol. Biol. Rep. 39: 7183-7192, 2012.

Shan, H., Chen, S.M., Jiang, J.F., Chen, F.D., Chen, Y., Gu, C.S., Li, P.L., Song, A.P., Zhu, X.R., Gao, H.S., Zhou, G.Q., Li, T., Yang, X.: Heterologous expression of the Chrysanthemum R2R3-MYB transcription factor $C m M Y B 2$ enhances drought and salinity tolerance, increases hypersensitivity to ABA and delays flowering in Arabidopsis thaliana. - Mol. Biotechnol. 21: 160-173, 2012.

Stracke, R., Werber, M., Weisshaar, B.: The R2R3-MYB gene family in Arabidopsis thaliana. - Curr. Opin. Plant. Biol. 4: 447-456, 2001.

Szabados, L., Savouré, A.: Proline: a multifunctional amino acid. - Trends Plant Sci. 15: 89-97, 2010.

Ullah, A., Qamar, M.T.U., Nisar, M., Hazrat, A., Rahim, G., Khan, A.H., Hayat, K., Ahmed, S., Ali, W., Khan, A., Yang, X.: Characterization of a novel cotton MYB gene, GhMYB108like responsive to abiotic stresses. - Mol. Biol. Rep. 47: 15731581,2020

Vannini, C., Campa, M., Iriti, M., Genga, A., Faoro, F., Carravieri, S., Rotino, G.L., Rossoni, M., Spinardi, A., Bracale, M.: Evaluation of transgenic tomato plants ectopically expressing the rice Osmyb4 gene. - Plant Sci. 173: 231-239, 2007.

Wang, J.Y., Li, Q., Mao, X.G., Li, A., Jing, R.L.: Wheat transcription factor TaAREB3 participates in drought and freezing tolerances in Arabidopsis. - Int. J. biol. Sci. 12: 257 269, 2016.

Weston, K.: MYB proteins in life, death and differentiation. Curr. Opin. Genet. Dev. 8: 76-81, 1998.

Wolfe-Simon, F., Starovoytov, V., Reinfelder, J.R., Schofield, O., Falkowski, P.G.: Localization and role of managaese superoxide dismutase in a marine diatom. - Plant. Physiol. 142: 1701-1709, 2006.

Wu, D.D., Sun, Y.H., Wang, H.F., Shi, H., Su, M.X., Shan, H.Y., Li, T.T., Li, Q.L.: The SlNAC8 gene of the halophyte Suaeda liaotungensis enhances drought and salt stress tolerance in transgenic Arabidopsis thaliana. - Gene 662: 10-20, 2018.

Wu, J.Y., Wong, K., Ho, K.P., Zhou, L.G.: Enhancement of saponin production in Panax ginseng cell culture by osmotic stress and nutrient feeding. - Enzyme Microbiol. Tech. 36: 133-138, 2005.

Yao, P.F., Sun, Z.X., Li, C.L., Zhao, X.R., Li, M.F., Deng, R.Y., Huang, Y.J., Zhao, H.X., Chen, H., Wu, Q.: Overexpression 
of Fagopyrum tataricum FtbHLH2 enhances tolerance to cold stress in transgenic Arabidopsis. - Plant Physiol. Biochem. 125: 85-94, 2018.

Yamagishi, M.: Isolation and identification of MYB transcription factors (MYB19Long and MYB19Short) involved in raised spot anthocyanin pigmentation in lilies (Lilium spp.). - J. Plant Physiol. 250: 153-164, 2020.

Yuan, Y., Shi, Y.M., Tang, D.Q.: Isolation and characterization of R2R3-MYB and basic helix-loop-helix (bHLH) transcription factors involved in anthocyanin biosynthesis in tulip tepals. Acta. Physiol. Plant. 32: 1-14, 2020.
Yue, P.Y., Mak, N.K., Cheng, Y.K., Leung, K.W., Ng, T.B., Fan, D.T., Yeung, H.W., Wong, R.N.: Pharmacogenomics and the yin/yang actions of ginseng: anti-tumor, angiomodulating and steroid-like activities of ginsenosides. - Chin. Med. 2: 6-18, 2007.

Zhao, Y.Y., Yang, Z.E., Ding, Y.P., Liu, L.S., Han, X., Zhan, J.J., Wei, X., Diao, Y.Y., Qin, W.Q., Wang, P., Liu, P.P., Sajjad, M., Zhang, X.L., Ge, X.Y.: Over-expression of an R2R3 MYB gene, GhMYB73, increases tolerance to salt stress in transgenic Arabidopsis. - Plant. Sci. 286: 28-36, 2019. 